\title{
Representações Mentais de Apego e Percepção de Práticas Parentais por Jovens Adultas
}

\author{
Mental Representations of Attachment and the Perception \\ of Parental Pratices by Young Adults
}

\author{
Vanessa Fonseca Gomes* \& Cleonice Alves Bosa \\ Universidade Federal do Rio Grande do Sul
}

\begin{abstract}
Resumo
O objetivo deste estudo foi investigar se o conhecimento ou não do script de base segura (representações mentais de apego) por jovens adultas afeta as cognições que compõe as suas futuras práticas parentais. Participaram 60 universitárias, com idades entre 18 e 25 anos divididas em dois grupos (Grupo 1: conhecimento do script de base segura e Grupo 2: desconhecimento do script de base segura, avaliado por meio do Attachment Script Assessment). As práticas parentais foram analisadas através da observação de videoclipes sobre interações mãe-criança. Os resultados demonstraram que os dois grupos se distinguiram em apenas duas categorias de práticas parentais: Promoção de Independência e Atribuição de Causalidade ao Comportamento da Criança, confirmando parcialmente as expectativas do estudo. Palavras-chaves: Apego; Sociocognição; Práticas parentais.

Abstract

The aim of this study was to investigate whether mental representations of attachment affect the perception that young adults have while observing interactions of children and their mothers in a laboratory situation. Parental practices were defined as socio-cognitions (goals, plans, expectations and attributions) present during child rearing context. Those socio-cognitions were access through the observations of video-clips of mother-child interactions. Attachment was analyzed through the Attachment Script Assessment. The results showed that only two categories distinguished the two groups: Independence Development and Causal Attribution to Child Behavior, confirming partially the initial expectation of the study.

Keywords: Attachment; Socio-cognition; Parental practices.
\end{abstract}

Ter apego ou estar apegado é ter alguém como base segura preferencial para explorar o ambiente. A teoria do apego (Bowlby, 1973/1998) foi um empreendimento importante para compreender os efeitos das primeiras relações com os cuidadores primários no desenvolvimento socioemocional. O pressuposto central da teoria é que a qualidade do vínculo entre a criança e o cuidador primário possui importantes implicações para o desenvolvimento da autoconfiança, da auto-estima, da sociabilidade e de outros aspectos desenvolvimentais da criança. Pesquisas importantes nesta área mostraram (Sroufe, Carlson, \& Shulman, 1993) que crianças com apego seguro apresentam maior facilidade na resolução das tarefas do desenvolvimento pós-infância. Em contraste, crianças com apego inseguro tendem a apresentar maiores dificuldades em aspectos como independência, resi-

* Endereço para correspondência: Rua Gal. Couto de Magalhães, 1155, Apto. 601, Higienópolis, Porto Alegre, RS, Brasil, CEP 90540-131.E-mail: vanessafgomes@yahoo.com . Projeto parcialmente financiado pela Coordenação de Aperfeiçoamento de Pessoal de Nível Superior (CAPES). liência, competência social e saúde emocional (Urban, Carlson, Egeland, \& Sroufe, 1991). Para Sroufe, Egeland, Carlson e Collins (2005), o elemento central do comportamento de apego é a sua organização. Assim, o significado do comportamento é dado pela sua organização com outros comportamentos, considerando-se os aspectos do contexto e de outras pessoas envolvidas. Tal organização se expressa na interação com a emoção, com a cognição e com o comportamento social, sendo que o desenvolvimento é definido pelas mudanças desta organização ao longo do tempo, com importantes implicações na afirmação das diferenças individuais.

Thompson (1998) afirmou que as primeiras experiências infantis com os pais afetam as estratégias de criação de filhos. Contudo, a teoria do apego não oferece explicações de como ocorre tal fenômeno. Por exemplo, como as primeiras experiências com os cuidadores primários afetam a futura responsividade parental? Ou ainda, como as primeiras experiências dos pais com os seus próprios pais afetam as práticas de socialização? Diante desta lacuna, sugere-se um modelo alternativo, que leva em consideração os processos subjacentes que ligam as pri- 
meiras experiências com cuidadores primários e as futuras práticas parentais, integrando conceitos da teoria sociocognitiva (Bandura, 1999) e da teoria do apego.

$\mathrm{Na}$ última década, pesquisadores em psicologia do desenvolvimento têm dado muita atenção ao impacto das cognições parentais nas práticas de criação dos filhos (Bugental \& Happaney, 2005; Miller, 1995; Wilson, Gardner, Burton, \& Leung, 2007). Por cognições parentais entendem-se as metas, planos, expectativas e atribuições sobre o modo de criação de filhos. As metas parentais podem ser definidas como o resultado daquilo que os pais desejam alcançar na interação com os filhos (Gollwitzer \& Moskowitz, 1996; Hastings \& Grusec, 1998) e são eliciadas por situações específicas vividas durante a experiência de criação de filhos, variando de acordo com o tempo e o contexto.

Pesquisas sobre práticas parentais salientam a importância das metas, pois elas são um dos principais fatores que determinam o comportamento dos pais. Elas influenciam o processamento da informação, o modo como se vivenciam emoções e o curso de ação escolhido, exercendo grande influência na variação de comportamentos (Dix, 1992; Lagacé-Séguin \& Coplan, 2005). Além disso, elas atuam conjuntamente com outras cognições, servindo para organizar e guiar as interpretações causais do comportamento e do afeto da criança (Hastings \& Grusec, 1998). Contudo, pouco se conhece sobre os aspectos relacionados à natureza das diferenças individuais nas metas. Esse fato é relevante, pois evidências empíricas demonstram que as cognições parentais são preditoras das práticas de criação dos filhos (Bugental \& Happaney, 2005; Grusec \& Mammone, 1995).

Os planos, por sua vez, são os meios de tornar possível a realização das metas, enquanto que as expectativas parentais contêm idéias sobre os aspectos que os pais acreditam que seus filhos podem e devem alcançar durante o curso do desenvolvimento (Beek, Genta, Costabile, \& Sansavini, 2006). Conforme Bugental e Johnston (2000), as expectativas parentais podem ser consideradas como cognições descritivas, pois elas englobam o conhecimento que os pais possuem sobre seus filhos.

As atribuições parentais podem ser entendidas como avaliações que os pais fazem sobre o comportamento do filho (Bugental \& Happaney, 2005; Leung \& Slep, 2006). Nos últimos cinco anos, a maioria dos estudos nesta área focaliza os efeitos da atribuição parental nas práticas parentais dentro do contexto de grupos clínicos (ex: depressão, autismo, crianças com problemas de comportamentos, entre outros). Tais estudos revelaram que a atribuição parental exerce um papel fundamental, uma vez que ela prediz a escolha e adesão ao tratamento, constituindo um importante fator para ser trabalhado nos programas de intervenção com pais. Apesar destas evidências serem interessantes, permanecem controversos os resultados sobre o impacto das atribuições parentais na criação de filhos, durante o curso do desenvolvimento infantil considerado típico.
Ainda que a relação entre a qualidade do apego e as práticas parentais posteriores seja uma premissa da teoria do apego, faltam evidências sobre esta possível relação nesta área. Nesse sentido, este estudo busca testar este modelo alternativo, tentando esclarecer se a qualidade das primeiras experiências infantis afeta as cognições que compõem as futuras práticas parentais.

O objetivo deste estudo foi investigar se o conhecimento ou não do script de base segura (representações mentais de apego), por jovens adultas, afeta as cognições que constituirão as suas futuras práticas parentais. Considerando o pressuposto da teoria de que estas representações mentais estão presentes mesmo antes de qualquer experiência com crianças, será investigada a presença deste fenômeno em jovens adultas, isto é, que ainda não são mães. Espera-se que as jovens com representações mentais de apego seguro sejam mais responsivas e sensíveis às necessidades e aos desejos da criança. A sensibilidade materna seria expressa, ao mesmo tempo, através da importância que as jovens atribuem à exploração livre do ambiente, pela criança (autonomia) e pela sua disponibilidade em auxiliar a criança em qualquer eventualidade. Espera-se também que essas jovens sejam mais empáticas e atentas à perspectiva infantil e mais interessadas em auxiliar a criança no modo de interpretar e significar os acontecimentos.

\section{Método}

\section{Participantes}

Participaram 92 universitárias, das quais 60 foram escolhidas para compor a amostra, com base nos resultados do Attachment Script Assessment. As universitárias foram recrutadas através do "Banco de Sujeitos" ${ }^{1}$ da Universidade de Stony Brook. Estes alunos recebem créditos acadêmicos pela sua participação em pesquisas. Participantes com pouca fluência verbal na língua inglesa e com filhos foram excluídos da amostra.

\section{Materiais e Instrumentos}

Consentimento Livre-Esclarecido. Este documento informou os participantes sobre os objetivos e os procedimentos do estudo. Somente participaram da amostra, universitárias que registraram o consentimento em protocolo próprio.

Ficha de Dados Demográficos. Nesta ficha constaram informações sobre sexo, idade, número de irmãos, nacio-nalidade da participante, escolaridade e nacionalidade dos pais. Também foram coletadas informações sobre a experiência com crianças. Neste item, as participantes responderam 12 questões (em uma escala de 1 a 8 , sendo que 1 representou pouca ou nenhuma e 8 significou muita experiência) sobre o seu conheci- 
mento acerca de brincadeiras, cuidados infantis, bem como o ensinamento de habilidades e bons comportamentos a crianças.

Attachment Script Assessment ([ASA]; Waters \& Rodrigues, 2001). Este instrumento avalia se o indivíduo possui ou não o script de base segura. O material é constituído por três folhas de papel. Cada folha de papel contém uma lista de 12 ou 14 palavras (Ex. mãe, bebê, urso de pelúcia, perdido, encontrado), distribuídas em três ou quatro colunas. As palavras sugerem uma história e ainda dá algumas pistas para a elaboração de uma narrativa. Na instrução, pede-se que o sujeito conte uma história, utilizando as palavras da lista. Embora as palavras sejam previamente estabelecidas (para guiar a história prototípica), o indivíduo possui a liberdade para elaborar a narrativa que quiser. As histórias construídas pelo sujeito são posteriormente analisadas em termos de coerência, conhecimento e acesso do script de base segura. Pessoas com conhecimento do script de base segura constroem histórias consistentes, girando em torno do tema. Pessoas que não possuem o conhecimento do script de base segura também as constroem de forma consistente, porém não relacionadas ao script de base segura, abordando outros tópicos. O ASA foi analisado por dois juízes cegos quanto aos resultados das respostas obtidas nos videoclipes da interação mãe-criança. Cada juiz analisou independentemente as histórias. Para o resultado final, foi utilizada a média dos escores dados pelos dois juízes. A ordem da apresentação das histórias foi modificada de sujeito para sujeito a fim de controlar o efeito de uma história sobre a outra. A distribuição dos participantes nos dois grupos (com e sem script de base segura) ocorreu da seguinte forma: Grupo 1 (com o conhecimento do script de base segura, representado pelos 30 escores mais altos) e Grupo 2 (sem o conhecimento do script de base segura - escores mais baixos).

Videoclipes de Interação Mãe-Criança (Gomes \& Waters, 2005). Este instrumento avaliou a percepção das universitárias sobre diferentes práticas parentais e é composto por 9 videoclipes de mães interagindo com seus filhos em três situações: (a) Brincadeira livre em um parque; (b) Situação de "limpeza" - recolher os brinquedos e colocá-los na estante; (c) Resolução de problema (ex: a criança precisa pegar uma bolacha presa entre dois vidros, utilizando um bastão). Isso permitiu que metas, planos, expectativas e atribuições fossem acessadas indiretamente. Cada uma destas situações contém videoclipes de três crianças diferentes. Os 9 videoclipes têm duração total de aproximadamente 30 minutos. A aplicação é iniciada com um videoclipe de "aquecimento" como prática (situação de brincadeira livre com a mãe em uma sala) a fim de familiarizar o indivíduo com o procedimento (as verbalizações obtidas desta fase não foram analisadas). As participantes assistiram cada videoclipe, duas vezes consecutivas. Na segunda apresentação, as imagens foram congeladas em diferentes momentos da interação entre mãe e criança. Durante estes momentos foi feita a seguinte pergunta à participante "Faça de conta de que você é a mãe e este é o/a seu/sua filho/a. Eu gostaria que você me dissesse o que estarias pensando naquele exato momento e porquê você estaria pensando aquilo". As imagens foram congeladas 58 vezes, através do botão de pausa do controle remoto, durante os 9 videoclipes e sinalizadas por uma estrela colorida no canto superior esquerdo da tela. Todos os participantes comentaram sobre os mesmos pontos nos vídeos, sendo as respostas gravadas em audioteipe. Posteriormente as respostas foram transcritas pela pesquisadora e analisadas por meio de análise de conteúdo quantitativa de modelo misto, no qual as categorias foram estabelecidas a priori (metas, planos, expectativas e atribuições) e as subcategorias foram geradas a partir dos dados obtidos. Para a distribuição das respostas em categorias e subcategorias, recorreuse a dois juízes que se mantiveram cegos quanto ao status de apego dos participantes. A própria pesquisadora e três bolsistas de iniciação científica participaram na análise das entrevistas. Devido à imensa quantidade de material, as verbalizações foram divididas em três partes, sendo que cada juiz codificou um terço das entrevistas. Apenas a pesquisadora analisou todo o material, participando de todo o processo. $\mathrm{O}$ agrupamento das categorias, pelos juízes, foi realizado inicialmente de forma independente, sendo a categorização final obtida através de consenso entre a pesquisadora e a bolsista. Um terceiro juiz participava do processo, na existência de dúvidas quanto à classificação das categorias. A ordem da apresentação das três situações foi alterada de sujeito para sujeito, controlando-se o efeito de um videoclipe sobre o outro.

Os escores foram computados a partir da ocorrência ou não de cada categoria, para cada participante. Desta forma, não foi considerado o número de vezes que tal categoria foi mencionada pela universitária. Por exemplo, mesmo que a participante verbalizasse a categoria "Disciplina/Obediência" cinco vezes durante a entrevista, ela recebeu apenas um ponto. Posteriormente, foram feitas distribuições de freqüências da categoria, nos dois grupos, as quais foram analisadas pelo z-teste para proporções a fim de se verificar possíveis diferenças, utilizando-se o programa estatístico chamado "Statistica"

\section{Procedimentos}

A administração dos instrumentos foi realizada individualmente, pela pesquisadora no laboratório de Apego na Universidade de Stony Brook e levou aproximadamente duas horas. Primeiramente, foi acessado o Attachment Script Assessment. Logo após, a aluna respondia o questionário sociodemográfico e, por fim, assistia aos vídeos clipes sobre a interação entre mães e filhos. 


\section{Resultados}

\section{Dados Sociodemográficos das Participantes}

A Tabela 1 mostra que a idade das universitárias variou entre 18 e 25 anos, não havendo diferenças estatísticas entre os dois grupos $(t=-0,26, g l=58, p=0,70)$. Optou-se por incluir apenas mulheres porque há evidências de que o relacionamento entre mãe-criança prediz maior variância nos resultados de estudos sobre desen- volvi-mento infantil (Barnard \& Solchany, 2005). Além disso, os instrumentos utilizados retratam as interações mãe-criança e não a de pais. A média de anos vividos nos EUA pelas universitárias foi de $17,8(D P=3,6)$, sendo o mínimo de 7 e o máximo de 25 anos. Não houve diferenças significativas entre os grupos nas variáveis (nacionalidade; $\chi^{2}=1,08, g l=1, p=0,29$, média de anos vividos nos EUA; $t=1,71, g l=58, p=0,09$ e experiência com crianças; $t=0,26, g l=58, p=0,97$ ) (Tabela1).

Tabela 1

Características Sociodemográficas no Grupo de Universitárias com o Script de Base Segura e o Grupo de Universitárias Sem o Script de Base Segura*

\begin{tabular}{|c|c|c|}
\hline Característica & $\begin{array}{l}\text { Presença do Script de Base Segura } \\
\qquad(n=30)\end{array}$ & $\begin{array}{l}\text { Ausência do Script de Base Segura } \\
\qquad(n=30)\end{array}$ \\
\hline \multicolumn{3}{|l|}{ Idade: } \\
\hline Média & 19,23 & 19,37 \\
\hline Desvio Padrão & 1,75 & 1,73 \\
\hline \multicolumn{3}{|c|}{ Experiência com crianças** } \\
\hline Média & 4,85 & 4,84 \\
\hline Desvio Padrão & 24,04 & 24,08 \\
\hline \multicolumn{3}{|c|}{ Anos vividos nos EUA } \\
\hline Média & 18,60 & 17,00 \\
\hline \multirow[t]{2}{*}{ Desvio Padrão } & 2,41 & 4,51 \\
\hline & $f(\%)$ & $f(\%)$ \\
\hline \multicolumn{3}{|l|}{ Posição na Família } \\
\hline Filho único & $2(6,7)$ & $1(3)$ \\
\hline Irmão Gêmeo & $1(3)$ & 0 \\
\hline Primogênito & $12(40)$ & $8(26,7)$ \\
\hline Filho do meio & $11(37)$ & $11(37)$ \\
\hline Filho mais novo & $4(13,2)$ & $10(33,3)$ \\
\hline
\end{tabular}

Nota. *Para as variáveis idade, anos vividos nos EUA, experiência com crianças foi usado o teste $t$. Para as variáveis nacionalidade e posição na família foi usado o teste Qui-quadrado. ** As participantes responderam questões em uma escala de 1 a 8 , sendo que 1 representou pouco ou nenhuma experiência e 8 significou muita experiência com crianças.

A Tabela 2 apresenta os dados sociodemográficos da família. Com relação à nacionalidade, verificou-se que a maioria das mães e dos pais das universitárias são norteamericanos. Os dados não revelaram diferenças significativas entre os grupos quanto à nacionalidade materna $\left(\chi^{2}=3.72, g l=3, p=0,29\right)$ e paterna $\left(\chi^{2}=6,67, g l=4\right.$, $p=0,15)$. Também não foram encontradas diferenças estatisticamente significantes nos grupos quanto a média de anos de escolaridade materna $(t=0,15, g l=58, p=$ $0,88)$ e paterna $(t=-0,66, g l=58, p=0,50)$.

As entrevistas geraram um total de 2906 verbalizações, sendo que o grupo 1 apresentou 1045 e o grupo 2: 1051. A análise das diferenças dos percentuais obtidos pelos dois grupos, através do Z-teste para proporções (Tabela $3)$, revelou diferenças significativas apenas quanto a metas parentais que dizem respeito à Promoção de Independência $(z=0,12 p=0,04)$ e atribuiçõos que as participantes fizeram sobre os comportamentos das crianças apresentados nos videoclipes - Compreensão acerca dos Comportamentos da Criança $(z=-0,18, p=0,03)$. Isso significa que um maior número de universitárias com a presença de script de base segura (grupo 1) reportou metas relacionadas com a Promoção de Independência das Crianças do que o grupo de universitárias sem o script de base segura (grupo 2). O mesmo resultado foi observado em relação à categoria Compreensão de Causalidade acerca dos Comportamentos da Criança, sendo que no grupo 1 a frequiência desta categoria foi maior do que no grupo 2. A mesma tendência foi observada quanto a categoria Preocupação com a Segurança da Criança, apesar da diferença entre os grupos não ter alcançado significância estatística. Contudo, esta diferença também não foi significativa estatisticamente. As categorias e subcategorias geradas a partir das análises das entrevistas nos grupos com (G1) e sem script de base segura (G2) são apresentadas na Tabela 3. 
Tabela 2

Distribuição da Escolaridade Materna e Paterna no Grupo e Grupo 2*

\begin{tabular}{lcccc}
\hline Característica & $\begin{array}{r}\text { Presença do Script de Base Segura } \\
(n=30)\end{array}$ & Ausência do Script de Base segura \\
& Mãe & Pai & Mãe & Pai \\
\hline Escolaridade & & & & \\
$\quad$ Média de anos & 14,37 & 14,07 & 14,27 & 14,53 \\
Desvio Padrão & 2,41 & 2,66 & 2,72 & 2,75 \\
& $f(\%)$ & $f(\%)$ & $f(\%)$ & $f(\%)$ \\
Fundamental incompleto & - & - & $1(3,3)$ & - \\
Fundamental & $1(3,3)$ & - & - & $2(6,6)$ \\
Médio incompleto & $1(3,3)$ & $4(13)$ & $1(3,3)$ & $1(3,3)$ \\
Médio & $6(20)$ & $7(23)$ & $8(26,6)$ & $7(23)$ \\
Superior incompleto & $9(30)$ & $6(20)$ & $5(16)$ & $3(10)$ \\
$\quad$ Superior & $13(43)$ & $13(43)$ & $15(50)$ & $17(56,6)$ \\
Nacionalidade & & & & \\
$\quad$ Norte-americanos & $19(63)$ & $19(63)$ & $12(40)$ & $12(40)$ \\
Centro-americanos & $3(10)$ & $3(10)$ & $3(10)$ & $3(10)$ \\
Sul-americanos & $1(3)$ & 0 & $1(3)$ & $2(7)$ \\
Asiáticos & $4(13)$ & $4(13)$ & $8(27)$ & $8(27)$ \\
Europeus & $3(10)$ & $4(13)$ & $3(10)$ & $2(7)$ \\
África & 0 & 0 & $3(10)$ & $3(10)$ \\
\hline
\end{tabular}

Nota. *Para as variável nacionalidade utilizou-se o teste Qui-quadrado. Para a variável escolaridade, utilizou-se a estatística descritiva e o teste $t$ para comparar a diferença entre a média de anos de estudo.

Tabela 3

Estrutura Geral das Categorias e Subcategoria

\begin{tabular}{lcc}
\hline Categorias & $\begin{array}{c}\text { Apego Seguro } \\
f(\%)\end{array}$ & $\begin{array}{c}\text { Apego Inseguro } \\
f(\%)\end{array}$ \\
\hline Metas & & \\
1. Disciplina/ Obediência & $66 \%(20)$ & $76 \%(23)$ \\
2. Promoção de Independência & $63 \%(19)^{*}$ & $37 \%(11)^{*}$ \\
3. Ensinar habilidades à criança & $56 \%(17)$ & $63 \%(19)$ \\
4. Preocupação com segurança da criança & $57 \%(17)$ & $37 \%(11)$ \\
5. Respeitar o ritmo da criança ao realizar determinada tarefa & $33 \%(10)$ & $37 \%(11)$ \\
6. Metas centradas nos pais & $40 \%(12)$ & $43 \%(13)$ \\
7. Regulação da Emoção & $66 \%(20)$ & $56 \%(17)$ \\
Planos & & \\
8. Oferecer estrutura para a realização da tarefa & $93 \%(28)$ & $93 \%(28)$ \\
9. Brincar com a criança & $37 \%(11)$ & $50 \%(15)$ \\
10. Monitoramento do comportamento da criança & $100 \%(30)$ & $96 \%(29)$ \\
11. Criticismo dos planos utilizados pelas mães nos videoclipes & $53 \%(16)$ & $66 \%(20)$ \\
Expectativas & & \\
12. Expectativas positivas em relação comportamento e características da criança & $63 \%(19)$ & $66 \%(20)$ \\
13. Expectativas negativas em relação comportamento e características da criança & $60 \%(18)$ & $66 \%(20)$ \\
Atribuições & & \\
14. A criança está distraída com brinquedos & $80 \%(24)$ & $87 \%(26)$ \\
15. A criança não entende o que está sendo solicitado & $63 \%(19)$ & $77 \%(23)$ \\
16. A criança está ignorando as ordens da mãe & $60 \%(18)$ & $63 \%(19)$ \\
17. Atribuição positiva sobre as características da criança & $63 \%(19)$ & $63 \%(19)$ \\
18. Atribuição negativas sobre as características da criança & $76 \%(23)$ & $73 \%(22)$ \\
21. Análise do comportamento da criança & $57 \%(17)^{*}$ & $30 \%(9)^{*}$ \\
\end{tabular}

Nota. $* p<0,05$. 


\section{Discussão}

Os resultados demonstraram que apenas duas categorias de práticas parentais distinguiram os dois grupos de jovens com e sem script de base segura: Metas de Promoção de independência e Compreensão de Causalidade acerca dos Comportamentos da Criança. Em relação à primeira, o grupo com script de apego seguro apresentou maior frequiência de metas no sentido de promover independência nos filhos, durante a interação mãe-criança. Esta categoria refletiu a capacidade de pensar no futuro da criança, além do desejo de incentivar autonomia, por acreditar que isto beneficia o desenvolvimento infantil. De acordo com Bowlby, o histórico de cuidados eficientes de uma determinada pessoa, gera a idéia de que a figura de apego estará sempre disponível, e de que ela terá o poder e a sabedoria de "salvá-la" de situações ameaçadoras, além de restaurar o seu equilíbrio durante a exploração do ambiente (Bowlby, 1973/1998).

Nesse sentido, pode-se pensar que a meta de promover independência é consistente com um dos aspectos mais importantes no fenômeno de base segura - encorajar a exploração da criança, ao mesmo tempo em que se oferece o apoio necessário. Ou seja, através da promoção de habilidades que estimulem autonomia na criança. Com isso, espera-se que a criança se desenvolva e que seja capaz de se aventurar sem receios, pois ela sabe que poderá contar com a sua figura de apego, quando necessário. Outro aspecto importante é que a teoria do apego implica a noção de que a segurança no apego prediz autoconfiança, sociabilidade e adequada auto-estima (Solomon \& George, 1999; Thompson, 1998). Neste sentido, incentivar a autonomia envolve também uma aposta nas capacidades da criança - condição básica para a emergência do senso de autoconfiança e de auto-estima elevada. Para Aviezer, Sagi, Resnick e Gini (2002), crianças com apego seguro possuem mais recursos para atividade exploratória, pois desenvolvem uma autonomia que as permite buscar novas informações necessárias na resolução de problemas.

Quanto à categoria Compreensão de Causalidade dos Comportamentos da Criança, o grupo de universitárias com o script de base segura demonstrou maior preocupação em entender os comportamentos da criança. Neste caso, notou-se que as participantes estavam mais atentas aos comportamentos expressos pelas crianças. Elas tentavam dar um significado ao que estava acontecendo nas interações mãe-criança observadas nos videoclipes. Esta reação englobou verbalizações do tipo "Eu estaria pensando... porque ela está tão interessada naquele brinquedo" ou "Será que ela não entende ou será que ela não está me escutando?", nas quais está clara a intenção de atribuir causalidade aos eventos.

Este comportamento pode constituir um indicador de sensibilidade materna, fator fundamental para teoria de apego. Estes resultados são consistentes com a idéia de que as representações mentais de apego afetam o nível de responsividade e sensibilidade materna aos sinais da criança, sendo fundamental para a formação dos vínculos afetivos (van Ijzendoorn, 1995). Pais com apego seguro tendem a responder mais adequadamente aos comportamentos da criança, quando comparados a pais com apego inseguro e assim tendem a criar crianças com apego seguro. Uma meta análise conduzida por van Ijzendoorn (1995) demonstrou que os padrões de apego dos pais influenciam os seus comportamentos nas interações com os seus filhos, o que por sua vez, afeta os padrões de apego de suas crianças. De acordo com o autor, a responsividade ou sensibilidade do cuidador é o fator responsável pela transmissão transgeracional de apego. De maneira semelhante, Fonagy, Steele e Steele (1991) afirmaram que mães com apego seguro são mais sensíveis do que mães com apego inseguro devido à capacidade de auto-reflexão, o que lhes auxilia a perceber o ponto de vista de seus filhos, sendo mais empáticas.

Esses achados estão de acordo com a noção de que as primeiras experiências com os cuidadores primários afetam as futuras cognições, afeto e comportamentos (Bakermans-Kranenburg, 2006; Waters \& Waters, 2006). Interessante ressaltar que tanto a meta de promover independência quanto a de compreensão de causalidade dos comportamentos da criança, constituem elementos chaves para a formação do relacionamento de apego. Conforme Leekers e Crockenberg (2006), o contexto de sensibilidade materna é fundamental para o desenvolvimento da autonomia, competência social e adaptação emocional. Isto demonstra que estes fatores atuam conjuntamente na formação da segurança de apego.

Os resultados também estão consistentes com o modelo proposto por Bakermans-Kranenburg (2006), em que as representações mentais de apego, que coordenam o script de base segura/insegura, predizem o tipo de práticas parentais. Pessoas que receberam o suporte de base segura de maneira coerente e consistente apresentarão o conhecimento deste script e, portanto, terão acesso aos seus elementos durante interações com outros que são significativos para elas. O script será ativado por eventos e informações relevantes, provocando determinadas reações comportamentais. Uma vez consolidado, o script influenciará as expectativas presentes em relacionamentos íntimos, através da organização das narrativas de apego e da seleção dos conteúdos relevantes.

A carência de relações entre apego e o restante das sociocognições (metas, planos, atribuições), neste estudo, contradiz a escassa literatura nesta área, o que pode, entre outros fatores, estar relacionado às limitações metodológicas desta pesquisa.

Uma avaliação crítica do método levantou questões referentes ao instrumento utilizado. Este estudo se distingue de outros já realizados sobre este tema, quanto à forma de acessar as cognições parentais. As metas, planos, expectativas e atribuições foram gerados a partir de relatos espontâneos das universitárias. Outras in- 
vestigações (Hastings \& Grusec, 1998; Leerkes \& Crockernberg, 2006) forneciam categorias a priori, solicitando que as participantes escolhessem o tipo de cognição que melhor representasse a sua realidade. Considerando que os scripts operam fora da consciência, pensa-se que a metodologia adotada é mais apropriada para acessar estes processos. Entretanto, este potencial do instrumento constitui também a sua maior limitação. Como o instrumento contemplou uma diversidade de situações, nas quais as participantes avaliaram pontos (58) específicos durante a interação mãe-criança, as categorias geradas podem ter sido demasiadamente genéricas, falhando em detectar as diferenças entre os grupos. Assim é possível que eventuais diferenças se "dissolveram" em meio a categorias muito semelhantes e interdependentes. Estudos que encontraram relações entre apego e o modo de criação de filhos, focalizaram comportamentos específicos como disciplina maternal ou situações extremas, como estresse infantil. Tais pesquisas compararam a maneira com que pessoas com apego seguro e inseguro atingem a meta de obter disciplina ou respondem ao estresse infantil. Este estudo mostrou apenas que os dois grupos não diferem quanto à intenção de obter obediência e disciplina. Embora as categorias tenham sido geradas a partir de uma abordagem qualitativa, os dados foram tratados quantitativamente, sendo que os resultados apenas nos indicaram se os grupos diferiram quanto à frequiência com que uma determinada cognição foi mencionada.

Com isso, conclui-se que mais ênfase deva ser dada à forma com que as cognições são transformadas em ação. Um dado interessante é que não houve diferenças entre os grupos quanto ao número de verbalizações apresentadas (o total de verbalizações analisadas foi 2906 , sendo que o grupo 1 teve: 1045 e o grupo 2: 1051). Isto demonstra, novamente, que os grupos não diferiram na quantidade de vezes em que mencionaram determinados tipos de cognições, mas que as diferenças residem na qualidade das interações mãe-criança. O próximo estudo deve focalizar a maneira com que futuros pais alcançam determinadas práticas parentais, assim como refinar o sistema de concordância entre juízes. $\mathrm{O}$ uso de cálculos de percentuais de concordância, ao invés de consenso, talvez auxilie nesta questão.

Outra limitação do estudo concentra-se no fato de que foram entrevistadas estudantes universitárias que ainda não eram mães. Conforme a teoria, as representações mentais de apego estão presentes, mesmo antes de qualquer experiência com crianças. Contudo, os resultados demonstraram que devemos levar em consideração o papel da experiência nas práticas parentais, pois este fator pode estar associado à ausência de diferenças encontradas entre os grupos. Azar, Nix e Makin-Byrd (2005) afirmaram que os scripts parentais oferecem o ponto de partida no processamento da informação. Todavia, não estão incluídos nestes scripts a capacidade executiva de funcionamento. Em outras palavras, a presença de conhecimento sobre modo de criação de filhos não está relacionada com o uso destas informações de maneira eficaz. As características do script apenas determinam se a capacidade executiva será ativada ou não. Em vista destes resultados, notou-se a necessidade de incluir experiência com práticas parentais no modelo.

Além disso, deve-se ressaltar que a noção do script de base segura ainda é incipiente e que mais estudos necessitam ser conduzidos para consolidar a idéia de que o script é, de fato, representativo das relações iniciais de apego e de seu padrão de segurança ou insegurança. É possível que o uso de critérios mais rígidos, que envolvesse maior contraste nos escores, possibilitasse a diferenciação nos padrões de base segura dos dois grupos.

$\mathrm{O}$ estudo foi pioneiro em investigar a forma com que o script afeta ou não as sociocognições presentes nas práticas parentais, apontando alternativas para investigações adicionais nesta área. Os resultados obtidos neste estudo não permitem responder se a falta de relação entre os domínios investigados é uma questão apenas metodológica ou também conceitual. De qualquer modo, a concepção de que a teoria sociocognitiva pode oferecer ferramentas para entender de que forma as representações mentais de apego afetam as futuras práticas de criação de filhos permanece como uma área de investigação promissora.

\section{Referências}

Aviezer, O., Sagi, A., Resnick, G., \& Gini, M. (2002). School competence in young adolescence: Links to early attachment relationships beyond concurrent self-perceived competence and representations of relationships. International Journal of Behavioral Development, 26, 397-409.

Azar, S., Nix, R., \& Makin-Byrd, K. (2005). Parenting schemas and the process of change. Journal of Marital and Family Therapy, 31, 45-58.

Bakermans-Kranenburg, M. J. (2006). Script-like attachment representations: Step towards a secure base for further research. Attachment \& Human Development, 8, 275-281.

Bandura, A. (1999). A social cognitive theory of personality. In L. Pervin \& O. John (Eds.), Handbook of personality (pp. 154-196). New York: Guilford.

Barnard, K., \& Solchany, J. (2005). Mothering. In M. Burnstein (Ed.), Handbook of parenting: Children and parenting (Vol. 3, pp. 3-26). New York: Lawrence Erlbaum.

Beek, V., Genta, M. L., Costabile, A., \& Sansavini, A. (2006). Maternal expectations about infant development of pre-term and full-term infants: A cross-national comparison. Infant and Child Development, 15, 41-58.

Bowlby, J. (1998). Apego e perda: Vol 2. Separação. São Paulo, SP: Martins Fonte. (Original work published 1973)

Bugental, D., \& Happaney, K. (2005). Parental Attributions. In M. Burnstein (Ed.), Handbook of Parenting: Children and Parenting (Vol 3, pp. 509-535). New York: Lawrence Erlbaum.

Bugental, D., \& Jonhston, C. (2000). Parental and child cognitions in the context of the family. Annual Review of Psychology, 51, 315-344. 
Dix, T. H. (1992). Parenting on behalf of the child: Empathic goals in the regulation of responsive parenting. In I. E. Sigel, A. V. McGillicuddy-DeLisi, \& J. J. Goodnow (Eds), Parental belief system: The psychological consequences for children (Vol 2, pp. 319-346). Trenton, NJ: Lawrence Erlbaum.

Fonagy, P., Steele, H., \& Steele, M. (1991). Maternal representations of attachment during pregnancy predict the organization of infant-mother attachment at one year of age. Child Development, 62, 891-905.

Gollwitzer, P., \& Moskowitz, G. (1996). Goal effects on action and cognition. In T. Higgins \& A. Kruglanski (Eds.), Handbook of social psychology (pp. 361-399). New York: Guildford Press.

Gomes, V., \& Waters, E. (2005). Videoclipes de interação mãecriança. Unpublished manuscript. Department of Health and Social Psychology, State University of New York.

Grusec, J., \& Mammone, N. (1995). Features and sources of parent's attributions about themselves and their children. In $\mathrm{N}$ Eisenberg (Ed.), Review of personality and social psychology (pp. 49-73). Thousand Oaks, CA: Sage.

Hastings, P., \& Grusec, J. (1998). Parenting goals as organizers of responses to parent-child disagreement. Developmental Psychology, 34, 465-479.

Lagacé-Séguin, D., \& Coplan, R. (2005). Maternal emotional styles and child social adjustment: Assessment, correlates, outcomes and goodness of fit in early childhood. Social Development, 14, 613-636.

Leerkes, E., \& Crockenberg, S. (2006). Antecedents of mothers' emotional and cognitive responses to infant distress: The role of family, mother, and infant characteristics. Infant Mental Health Journal, 27, 405-428.

Leung, D. W., \& Slep, A. M. S. (2006). Predicting inept discipline: The role of parental depressive symptoms, anger, and attributions. Journal of Consulting and Clinical Psychology, 74(3), 524-534.

Miller, S. (1995). Parents' attributions for their children's behavior. Child Development, 66, 1557-1584.

Solomon, J., \& George, C. (1999). The measurement of attachment security in infancy and childhood. In J Cassidy \& P Shaver (Eds.), Handbook of attachment: Theory, research and clinical aplications (pp. 287-316). New York: The Guildford Press.

Sroufe, L. A., Carlson, E., \& Shulman, S. (1993). Individuals in relationships: Development from infancy through adolescence. In D. C. Funder, R. Parke, C. Tomlinson-Keesey, \& K. Widaman (Eds.), Studying lives through time: Approaches to personality and development (pp. 315-342). Washington, DC: American Psychological Association.

Sroufe, L. A., Egeland, B., Carlson, E., \& Collins, A. (2005). The development of the person. New York: The Guildford Press.

Thompson, R. A. (1998). Early sociopersonality development. In N. Eisenberg (Ed.), Social, emotional, and personality development (Vol. 3, pp. 25-104). New York: Wiley.

Urban, J., Carlson, E., Egeland, B., \& Sroufe, L. A. (1991). Patterns of individual adaptation across childhood. Development and Psychopathology, 3, 445-460.

van Ijzendoorn, M. H. (1995). Adult representations, parental responsiveness, and infant attachment: A meta-analysis on the predictive validity of the adult attachment interview. Psychological Bulletin, 117, 387-403.
Waters, H. S., \& Waters, E. (2006). The attachment working models concept: Among other things, we build script-like representations of secure base experiences. Attachment \& Human Development, 8, 185-197.

Waters, H. S., \& Rodrigues, L. M. (2001). Are attachment scripts the building blocks of attachment representations: Narrative assessment of representations and the AAI. Paper presented at the biennial meeting of the Society for Research in Child Development. Minneapolis, MN. Retrieved September 9, 2005, from http://www.psychology.sunysb.edu/ attachment/srcd2001/HSWScripts/index.htm

Wilson, C., Gardner, F. Burton, J., \& Leung, S. (2007). Maternal attributions and observed maternal behaviour: Are they linked? Behavioural and Cognitive Psychotherapy, 2, 165178.
Recebido: 09/08/2007 $1^{a}$ revisão: $13 / 11 / 2007$ $2^{a}$ revisão: 03/06/2008 Aceite final: 09/07/2008 